\title{
Effect of Surrogate Surface Compliance on the Measured Stiffness of Snowboarding Wrist Protectors ${ }^{\dagger}$
}

\author{
Gemma Leslie ${ }^{1, *}$, Weizhuo Wang ${ }^{1}$, Keith Winwood ${ }^{1}$, Chris Liauw ${ }^{1}$, Nick Hamilton ${ }^{2}$ \\ and Tom Allen ${ }^{1}$ \\ 1 Faculty of Science and Engineering, Manchester Metropolitan University, Manchester M1 5GD, UK; \\ w.wang@mmu.ac.uk (W.W.); k.winwood@mmu.ac.uk (K.W.); christopher_M_liauw@outlook.com (C.L.); \\ t.allen@mmu.ac.uk (T.A.) \\ 2 Centre for Sports Engineering Research, Sheffield Hallam University, Sheffield S10 2LX, UK; \\ n.hamilton@shu.ac.uk \\ * Correspondence: gemma.e.leslie@stu.mmu.ac.uk; Tel.: +44-796-993-3631 \\ + Presented at the 13th conference of the International Sports Engineering Association, Online, \\ 22-26 June 2020.
}

Published: 15 June 2020

\begin{abstract}
Wrist injuries have been reported to account for 35\%-45\% of snowboarding injuries. Snowboarding wrist protectors are designed to limit impact forces and prevent wrist hyperextension. The absence of a standard for snowboarding wrist protectors makes it hard to identify models offering an adequate level of protection. Wrist surrogates are well suited for testing and benchmarking wrist protectors. This study investigated the effect of introducing a soft tissue simulant onto an otherwise stiff wrist surrogate on the bending stiffness of snowboarding wrist protectors. A compliant surrogate (stiff core and $3 \mathrm{~mm}$ thick silicone layer) and a comparable stiff surrogate were fabricated. Two snowboarding wrist protectors were tested on each surrogate, under three strapping conditions, following a bend test to $\sim 80^{\circ}$ wrist extension. The introduction of a compliant layer to the wrist surrogate gave higher torque values for a given wrist extension angle, increasing protector effective stiffness, relative to a rigid surrogate.
\end{abstract}

Keywords: wrist surrogate; biofidelic; snowboarding; wrist protection; injury prevention

\section{Introduction}

There are an estimated 10 to 15 million snowboarders worldwide [1]. Snowboarding injury risk has been reported to range from 0.8 to 8.0 injuries per 1000 snowboarding days, with $35 \%-45 \%$ of injuries reported to be to the wrist [1]. A distal radius fracture is the most common wrist injury amongst snowboarders, resulting from a compressive load applied to a hyperextended (exceeding normal range of motion) wrist [1-3]. Beginners and inexperienced snowboarders have a higher risk of wrist injury [4], possibly due to them putting out their hands in an attempt to cushion a fall. Wrist protectors are available to snowboarders, and are typically designed to limit impact forces (via palmer padding) and prevent wrist hyperextension (via palmer/dorsal splints) [1]. As a standard for snowboarding wrist protectors BS EN ISO 20320:2020 [5] has only just been published, it is currently difficult to identify models offering an adequate level of protection.

Tests for assessing personal protective equipment (PPE), such as wrist protectors, typically utilise a surrogate or anvil to represent the human limb [6,7]. These surrogates often consist of simplified geometries and stiff materials that provide a basic representation of the human body [8], which is ideal for test houses to reduce complexity and potential variability. Wrist surrogates have been developed for a bend test to measure the stiffness of snowboarding wrist protectors [9,10], and one has been implemented into the new standard [5]. The typical construction of a wrist surrogate 
for a bend test is hand and forearm parts made from stiff materials (e.g. thermoplastic/thermoset polymers), connected at the wrist joint with a low-friction hinge. Surrogate design has been shown to influence the measured stiffness of wrist protectors [10]. A simplified geometric representation of a hand and forearm based on anthropometric data (surrogate B in [10] and implemented into [5]) provided less variation in measurements than, both a simpler hand and forearm shape [11] and a hand and forearm based on a laser scan of a human arm.

Improvements in sports surrogates to increase biofidelity (modeling the response of a human) is arguably crucial in the development, testing and certification of sports PPE [8]. However, there must be a balance between surrogate biofidelity and repeatability. A wrist surrogate utilising a soft tissue simulant could represent a better prediction of human response than one made solely from stiff material. The addition of compliant material to the surrogate in [5] could improve the fit of the protector and limit it from slipping during testing [10]. This study investigated the effect of introducing a compliant layer into the BS EN ISO 20320:2020 wrist surrogate on the mechanical response of snowboarding wrist protectors.

\section{Materials and Methods}

\subsection{Surrogate Fabrication}

A rigid surrogate (defined as made from stiff materials) and a compliant surrogate (defined as having a stiff core with a silicone outer layer) were fabricated. Both surrogates consisted of a hand and forearm connected with a low-friction hinge joint at the wrist, and two steel fingers, as per the dimensions of the medium surrogate in [5]. The surrogate hand and forearm parts were modelled in CAD (Solidworks, 2019, Dassault Systems, Waltham, USA) as per the drawing in the draft standard (as per 15-05-2018) [5]. Both surrogates were additively manufactured (Markforged X7) in Onyx filament (short carbon fibre reinforced polyamide). The rigid surrogate was fabricated as per the drawing in draft standard [5]. To develop the compliant surrogate, the following changes were made to the original CAD model. The loft-cut function was used to reduce the periphery of the hand and forearm by $3 \mathrm{~mm}$ to create a hand and forearm core, allowing for the incorporation of a silicone outer layer (Figure 1a). Five $3 \mathrm{~mm}$ diameter holes were added through the hand and forearm core to allow the silicone to achieve a mechanical bond. Additively manufactured moulds, based on the external geometry of the original surrogate hand and forearm, created $3 \mathrm{~mm}$ thick cavities for silicone to fill during fabrication (Figure $1 \mathrm{~b}$ part 2, 4, 5,6). The moulds had a $5 \mathrm{~mm}$ diameter hole (towards the bottom of the dorsal side) to allow the silicone to be inserted into the cavity (Figure $1 \mathrm{~b}$ part 7), four 3 $\mathrm{mm}$ diameter cylindrical supports (two on each side protruding into the cavity) to ensure the cores sat central in the cavity, and $6 \mathrm{~mm}$ diameter holes at each corner so the two halves could be bolted together around the surrogate core. The hinge joint region was not edited between surrogates, to maintain range of motion without influencing joint friction (Figure 1c part 11).

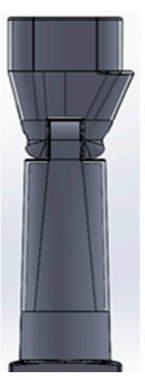

(a)

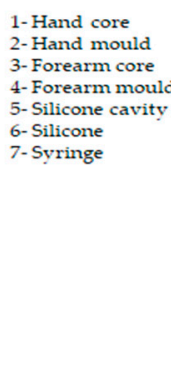

3- Forearm core 4- Forearm mould 6-Silicone y.ros

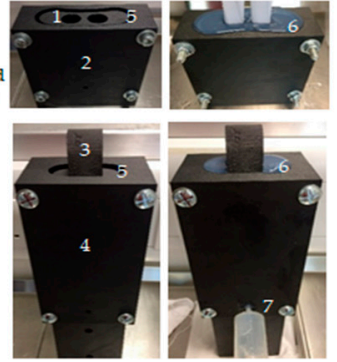

(b)
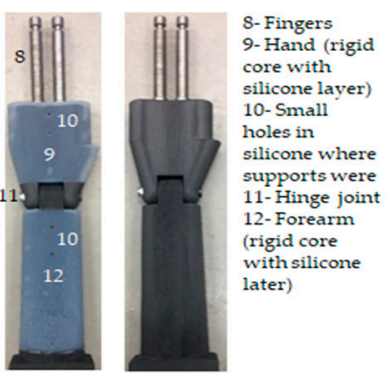

(c)

Figure 1. (a) Compliant surrogate CAD; (b) silicone moulding; (c) compliant surrogate (left) and rigid surrogate (right).

Maxillofacial silicone M511 (Technovent, Bridgend, UK) was selected as a candidate soft tissue simulant. M511 is a two-part platinum (vinyl addition) cure system, and has a Shore A hardness 
range of 15-20 once cured. Part A and part B were weighed (Salter 1036 BKSSDR, Kent, UK) in a 10:1 ratio, poured into a $500 \mathrm{~mL}$ mixing bowl, and vacuum mixed (FINOVAC 10423) for $120 \mathrm{~s}$ at room temperature $\left(20^{\circ} \mathrm{C}\right)$. The mixture was then transferred into a $10 \mathrm{~mL}$ syringe, and introduced to the silicone cavity via the hole in the mould (Figure $1 \mathrm{~b}$ part 7). The moulding was left for $24 \mathrm{~h}$ at room temperature to cure, then the surrogate was removed, and excess silicone along the mould joint was cut away with a Stanley knife. The fingers were inserted into the top of the hand, and the hand and forearm hinge joint were assembled. Component masses were as follows: rigid hand $84.1 \mathrm{~g}$, rigid forearm $268.7 \mathrm{~g}$, compliant hand $135.9 \mathrm{~g}$, compliant forearm $394.0 \mathrm{~g}$, fingers $308.2 \mathrm{~g}$, hinge joint fasteners $32.3 \mathrm{~g}$ (Mettler Toledo PE11, Leicester, UK). The difference in mass of the hand between the two surrogates resulted in a small, negligible difference of $0.025 \mathrm{Nm}$ in the torque produced at $90^{\circ}$ wrist extension.

\subsection{Bend Test}

Two snowboarding wrist protectors (both adult medium, right hand) were tested in a bend test $[9,10]$ to investigate if the addition of the compliant layer to the wrist surrogate affected their measured stiffness (short protector-Burton $\left(\right.$ and long protector-Flexmeter ${ }^{\mathrm{TM}}$ double sided). The surrogate was fastened to a bespoke rig with the forearm vertical and palm facing away from the uniaxial test machine (Hounsfield HK10S, Tinius Oslen, Surrey, UK; 1 kN load cell) (Figure 2). A cable connected the distal end of the surrogate fingers (via a $148 \mathrm{~g}$ clamp) to the test machine load cell via a pulley (Harken $22 \mathrm{~mm}$ micro block, Hampshire, UK) (Figure 2b). Positive vertical displacement of the load cell (test speed $-200 \mathrm{~mm} / \mathrm{min}$ ) applied an extension torque to the surrogate wrist via the cable, pulling the hand backwards (mimicking wrist extension) and bending the protector.

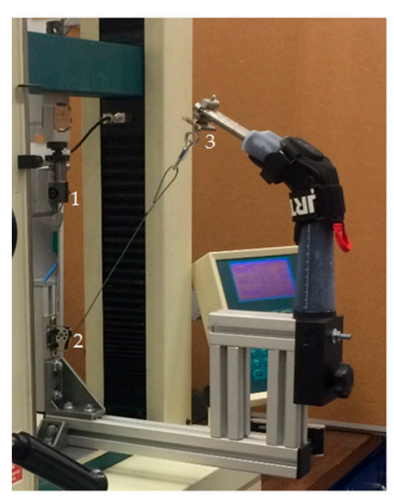

(a)

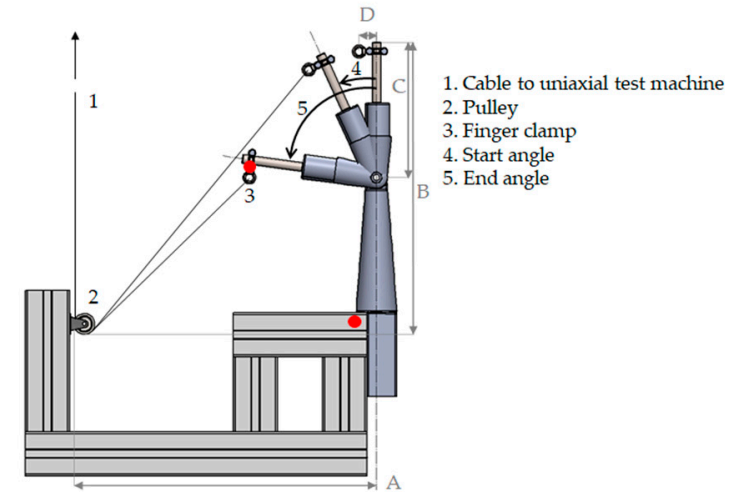

(b)

Figure 2. (a) Bend test setup, (b) schematic (A, B, C and D indicate manual measurements required for data analysis, and the two red dots indicate where markers were placed to track protector movement for video analysis).

Prior to testing and data collection, the linear displacement of the load cell required to give the desired final wrist extension of $\sim 80^{\circ}$ needed to be established, to prevent the surrogate reaching its extension limit of $90^{\circ}$. A used protector was fitted to the surrogate on the rig to determine the required load cell displacement $(136 \mathrm{~mm})$ to extend the surrogate wrist from its starting angle $\left(\sim 40^{\circ}\right.$ with finger clamp contributing a torque of $\sim 0.17 \mathrm{Nm}$ ) to $\sim 80^{\circ}$. Each test began by fitting the protector to the surrogate on the rig, connecting the finger clamp and cable and measuring the start angle of the wrist (Figure $2 \mathrm{~b}$ part 4). A linear displacement of the load cell of $136 \mathrm{~mm}$ was then applied, extending the wrist from the start angle to the end angle at $\sim 80^{\circ}$. Load and displacement were recorded at a sampling rate of $25 \mathrm{~Hz}$. The start and end angles of the wrist were measured for each trial with a digital inclinometer (PRO360, SPI, New York, NY, USA).

Three conditions were used for protector strap tightness [9]; loose, moderate and tight. Strap tightness was established for each condition by, (i) putting a protector on the surrogate, (ii) holding 
the surrogate horizontal, (iii) hanging either a 1, 2 or $3 \mathrm{~kg}$ mass from the straps of the protector, (iv) rotating the surrogate around its long axis to tighten the straps, and v) marking the position of the straps for future reference [10]. Tightness for each protector was set prior to testing using the marks established with the hanging masses. Six protectors of each style (short, long) were tested, with two of each tested at each strapping condition on each surrogate (three strapping conditions across two protector-surrogate pairings -24 different combinations). Three repeated tests were performed for each protector-surrogate-strapping combination ( $1 \mathrm{~min}$ rest period between repeats) (Total $=72$ tests). Protectors were defined as either: new (untested) or used (after 3 tests on a surrogate). Surrogates were alternated between combinations, so one new protector of each style was tested at each strapping condition on each surrogate (15 min recovery period between each test surrogate). Protectors were re-positioned and re-strapped between tests. The first test for each combination (24/72 tests) was filmed side on, to view wrist extension, with a camera (Phantom Miro R110, Vision Research UK Ltd., Bedford, UK) set at a resolution of $1280 \times 800$ and a capture rate of $24 \mathrm{~Hz}$. The camera was calibrated from an image taken of a ruler on the test rig. If protector slippage was clearly observed in a video, then the video frames were used to take measurements between a feature on the protector and a marker on the rig.

\subsection{Data Analysis}

Load and displacement values obtained were converted to torque and angle, respectively, using trigonometry (start and end angle, and manual measurements in Figure $2 \mathrm{~b}$ were required for this process) [9,10]. A Matlab (R2018a, Mathworks, Natick, MA, USA) script using the fit function with the nonlinear least-squares algorithm was used to curve fit and filter the torque vs. angle data. Statistical analysis was used to compare surrogates at each strapping condition (for each protector). The mean torque values at $75^{\circ}$ wrist extension (upper wrist extension range $[2,3]$ ) were compared using ANOVA (Analysis of variance), and post hoc analysis was carried out using Tukey's pairwise comparison at a confidence level of 95\%, using Minitab (v18 statistical software, Pennsylvania, PA, USA). Video observations found the dorsal side of the short protector slipped more on the rigid surrogate than the compliant surrogate (under moderate and tight strapping conditions).

\section{Results}

In most cases ( $86 \%, 31 / 36$ tests), the compliant surrogate gave higher torque values at $75^{\circ}$ wrist extension than the rigid surrogate (Figure 3). The five exceptions when the rigid surrogate gave higher torque values than the compliant surrogate occurred on repeated tests on the short protector, and four of these were on the third repeat for a given strapping condition. Significant differences at $75^{\circ}$ wrist extension $(p<0.05)$ occurred between the surrogates when fitted with the long protector (loose $p=0.005$, moderate $p=0.004$ and tight $p=0.021$ ). The torque required to extend the wrist to $75^{\circ}$ tended to decrease with repeated tests, indicating the stiffness of the protectors decreased with testing (Figure 3). Torque values increased with wrist extension (Figure 4), and in most instances $(31 / 32,97 \%)$ for the first test on both new and used protectors, the torque required to extend the wrist to $75^{\circ}$ increased with strap tightness (Figure 3). When analysing a range of wrist extension angles (Figure 4), the torque required to extend the wrist varied with strap tightness. A small torque $(<1 \mathrm{Nm})$ is displayed at $40^{\circ}$ (Figure 4), due to tension in the cable at the start of each test. The long protector gave higher torque values for a given wrist extension angle than the short protector (Figures 3 and 4), following the trend of previous research [9,10]. The torque values observed from [10] (equivalent to moderate strap tightness) fell between those reported here under moderate and tight strapping conditions on the rigid surrogate (shown by the red lines in Figure 4).

Measurements on the video frames (start and end angle) showed the top of the dorsal side of the short protector slipped $13 \mathrm{~mm}$ towards the fingertips when on the rigid surrogate, compared to $8 \mathrm{~mm}$ on the compliant surrogate (both new protectors under moderate strapping condition) (Figure 5). Slippage also occurred at the same location on the used short protectors under moderate strapping condition ( $9 \mathrm{~mm}$ rigid surrogate, $7 \mathrm{~mm}$ compliant surrogate), and the short protector under tight strapping condition (new protector $-14 \mathrm{~mm}$ rigid surrogate, $10 \mathrm{~mm}$ compliant surrogate, and used 
protector $-16 \mathrm{~mm}$ rigid surrogate, $7 \mathrm{~mm}$ compliant surrogate). While the short protector slipped in a similar manner under loose strapping condition, no clear differences were observed between the surrogates. The long protector was not observed to slip in any test.

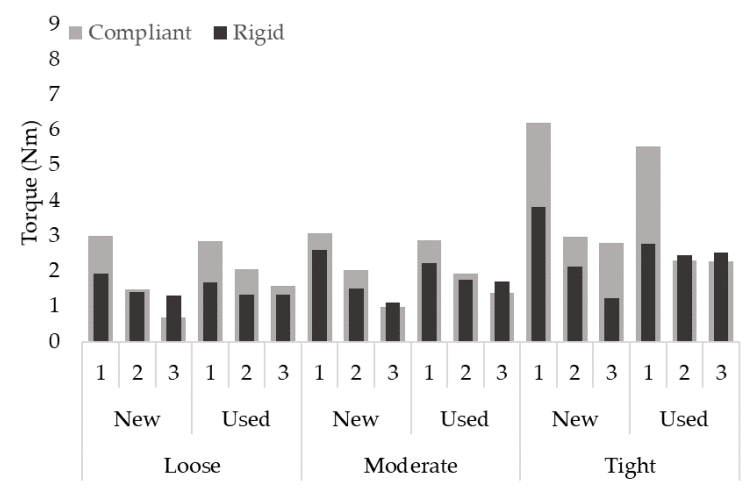

(a)

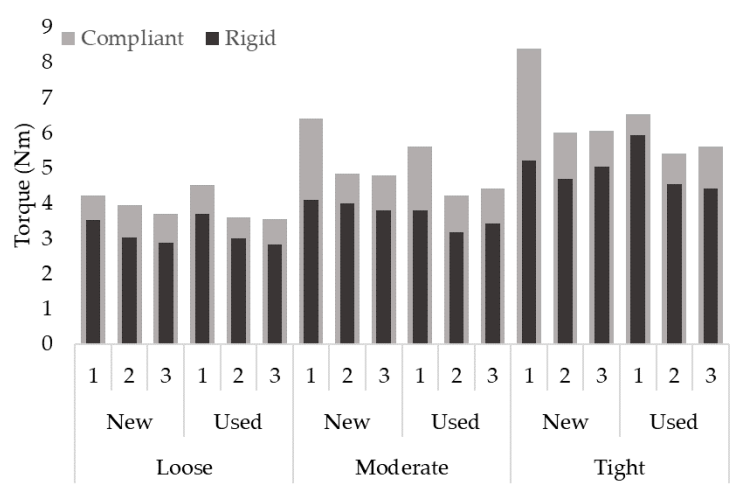

(b)

Figure 3. Torque at $75^{\circ}$ wrist extension for (a) short and (b) long protector. The $\mathrm{x}$-axis indicates the test repeat, protector condition, and strapping condition.

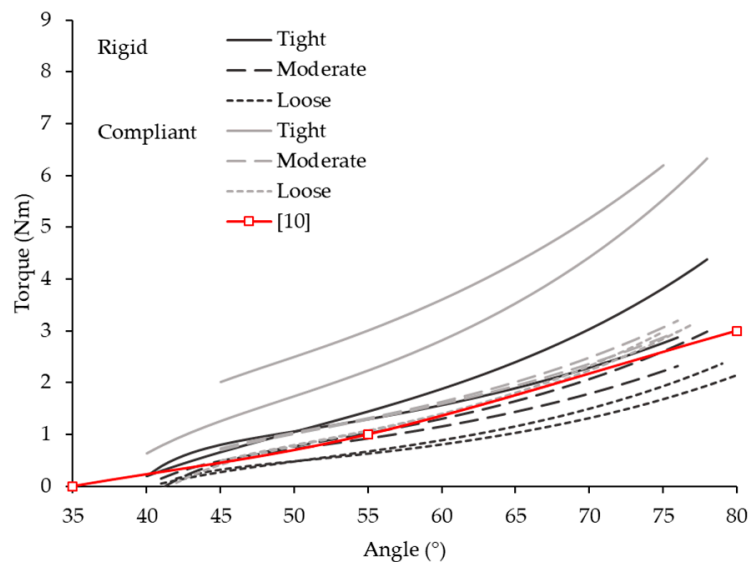

(a)

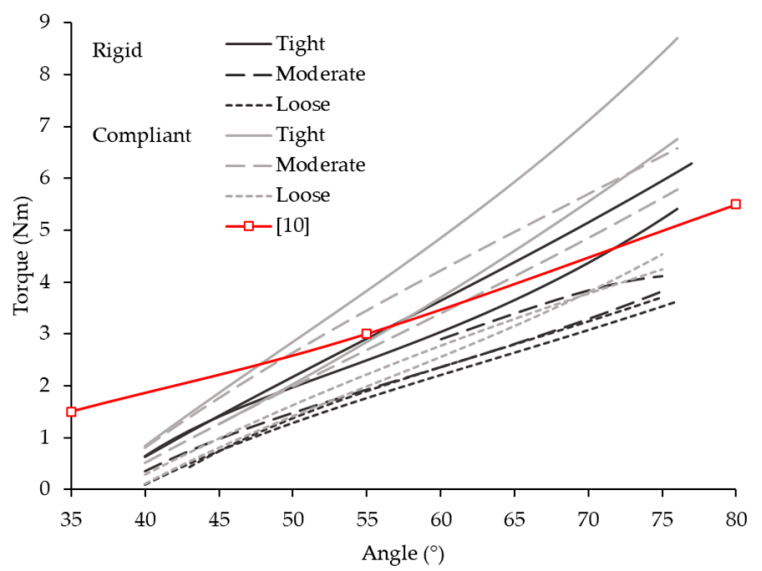

(b)

Figure 4. Torque vs. angle for test 1 of a new and used protector at each strapping condition (tightsolid line, moderate-broken line, loose-dotted line), for (a) short and (b) long protector, plotted alongside data (digitised from graph) from [10].

\section{Discussion}

In general, the compliant surrogate gave higher torque values for a given wrist extension angle for both protectors. At $75^{\circ}$ wrist extension, the compliant surrogate gave higher torque values in $86 \%$ (31/36) of tests (Figure 3). The difference in torque values between the surrogates may have been due to the silicone increasing the friction between the protector and surrogate, although this was not measured. Future work on surrogate design could try to control the protector-surrogate friction. Video analysis showed the top of the dorsal side of the short protector slipped (Figure 5) a mean average of $5 \mathrm{~mm}$ more during testing when fitted to the rigid surrogate under moderate and tight strapping conditions compared to the compliant surrogate. The reduced slippage of the short protector on the compliant surrogate may have caused the splints to engage more during testing, explaining the increased torque required to extend the wrist. It was unclear from the videos whether protector slippage occurred at other locations on the surrogates. Synchronized cameras viewing different regions of the protectors could enable more insight into protector slippage during bend testing. Strain gauges on protector splints could provide insight into how much they bend during testing on different surrogates. 


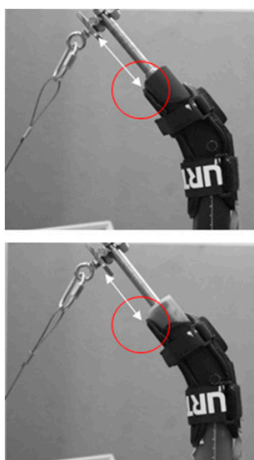

(a)

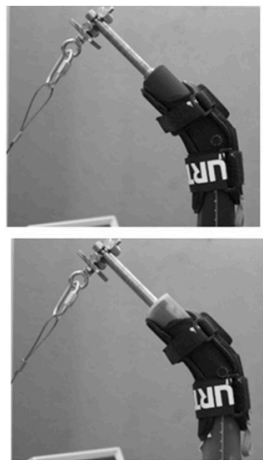

(b)

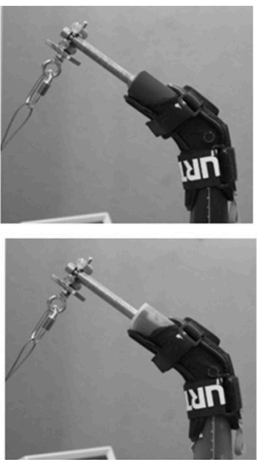

(c)

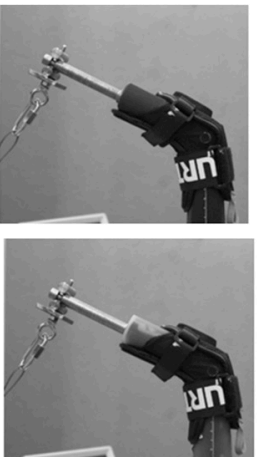

(d)

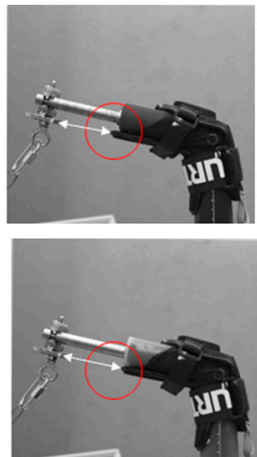

(e)

Figure 5. Video sequence at (a) $0 \mathrm{~s}$, (b) $10 \mathrm{~s}$, (c) $20 \mathrm{~s}$, (d) $30 \mathrm{~s}$, (e) $40 \mathrm{~s}$ of the short protector on the rigid (top) and compliant (bottom) surrogate. All of them are moderate strapping condition. Red circles highlight protector slippage. White line indicates measured distance.

The stiffness of protectors decreased during test repeats, indicating they degraded. The difference in torque value between the first and second (repeat) test of a new protector ranged from 0.5 to $3.2 \mathrm{Nm}$ (short protector) and 0.3 to $2.4 \mathrm{Nm}$ (long protector) at $75^{\circ}$ wrist extension (Figure 3). After resting the protectors appeared to have partially recovered, with differences in torque values between the first test of a new protector and the first test a used protector ranging from 0.2 to $1.0 \mathrm{Nm}$ (short protector) and -0.7 to $1.9 \mathrm{Nm}$ (long protector) at $75^{\circ}$ wrist extension. All of the second and third tests showed differences in torque values $<1 \mathrm{Nm}$, however, due to protectors degrading, these torque values are not a true indication of protector stiffness when new. New protectors, or rest periods before repeating testing, should be used in future tests comparing wrist surrogates, to limit the influence of protector degradation on results.

At $75^{\circ}$ wrist extension, the torque values for the short and long protector, under moderate strapping conditions, on the rigid surrogate were 2.6 and $4.1 \mathrm{Nm}$ respectively (Figure 3). Torque values of $\sim 2$ and $\sim 5 \mathrm{Nm}$ were reported at $75^{\circ}$ (based on line connecting points from graph [10]) for a short and long protector under moderate strapping conditions on the geometric surrogate in [10]. The torque values reported in [10] under moderate strapping conditions fell between those observed here for moderate and tight strapping conditions (Figure 4), indicating potential issues in achieving a set strap tightness between operators. An improved method providing increased control over protector strap tightness could include embedding of pressure sensors in the surrogate; the latter approach would have the added benefit of providing measurements of pressure at specific locations on the surrogate during testing.

Future work could further develop the surrogate by: reducing the mass of the finger clamp to reduce its effect on torque measurements and wrist start angle; reducing the mass of the fingers by additively manufacturing them with the hand as one part; the addition of a device to measure wrist angle during testing and enable the test machine start/end position to be determined by angle rather than displacement of the load cell. More protectors of the same type should be tested to determine protector variance and repeatability of the surrogates. Testing of other snowboarding wrist protector designs should be carried out in order to determine whether the compliant surrogate continues to cause increased effective stiffness of the protector. Future surrogate development could also assess the effect of different varieties of silicone elastomers and variation of silicone thicknesses on the effective stiffness of the protector.

\section{Conclusions}

The addition of a $3 \mathrm{~mm}$ thick compliant outer layer to a wrist surrogate gave higher torque values in a bend test for a given wrist extension angle, relative to an equivalent rigid surrogate. This observation indicated that the effective stiffness of the protector had increased. The torque required to extend the wrist on both a rigid and compliant surrogate increased with protector strap tightness. Significant differences in torque values at $75^{\circ}$ wrist extension were found between the compliant and 
rigid surrogate when a long protector was fitted using different strapping tightnesses. A short protector slipped more during testing when fitted to a rigid surrogate compared with a compliant surrogate.

Funding: This research was funded by Manchester Metropolitan University.

Acknowledgments: We would like to thank Manchester Metropolitan University for funding this research, the technicians for fabricating the rig, and Adil Imam for assistance during testing.

Conflicts of Interest: The authors declare no conflict of interest.

\section{References}

1. Michel, F.I.; Schmitt, K.U.; Greenwald, R.M.; Russell, K.; Simpson, F.I.; Schulz, D.; Langran, M. White Paper: Functionality and efficacy of wrist protectors in snowboarding-Towards a harmonized international standard. Sports Eng. 2013, 16, 197-210.

2. Shultz, S.J.; Houglum, P.A.; Perrin, D.H. Wrist and Hand. In Examination of Musculoskeletal Injuries, 3rd ed.; Stone, J.J., Matz, K., Myrick, S., Ed.; Human Kinetics: Champaign, IL, USA, 2015; pp. 319-353.

3. Levangie, P.K.; Norkin, C.C. The Wrist Complex. In Joint Structure and Function: A Comprehensive Analysis, 4th ed.; Davis Company: Philadelphia, FA, USA, 2000; pp. 305-352.

4. Maurel, M.L.; Fitzgerald, L.G.; Miles, A.W.; Giddins, G.E. Biomechanical study of the efficacy of a new design of wrist guard. Clin. Biomech. 2013, 28, 509-513.

5. ISO. BS ISO EN 20320:2020. Protective Clothing for Use in Snowboarding - Wrist Protectors-Requirements and Test Methods; ISO: Geneva, Switzerland, 2020.

6. BSI. BS EN 13061:2009. Protective Clothing. Shin Guards for Association Football Players. Requirements and Test Methods. British Standards Institution; BSI: London, UK, 2009.

7. BSI. BS 6183-3:2000. Protective Equipment for Cricketers. Leg Protectors for Batsmen, Wicket-Keepers and Fielders, and Thigh, Arm and Chest Protectors for Batsmen; BSI: London, UK, 2000.

8. Payne, T.; Mitchell, S.; Bibb, R.; Waters, M. Initial validation of a relaxed human soft tissue simulant for sports impact surrogates. Procedia Eng. 2014, 72, 533-538.

9. Adams, C.; James, D.; Senior, T.; Allen, T.; Hamilton, N. Development of a method for measuring quasistatic stiffness of snowboard wrist protectors. Procedia Eng. 2016, 147, 378-383.

10. Adams, C.; James, D.; Senior, T.; Allen, T.; Hamilton, N. Effect of surrogate design on the measured stiffness of snowboarding wrist protectors. Sports Eng. 2018, 21, 217-225.

11. BSI. BS EN 14120:2003+A1:2007. Protective Clothing - Wrist, Palm, Knee and Elbow Protectors for Users of Roller Sports Equipment-Requirements and Test Methods; BSI: London, UK, 2003.

(c) 2020 by the authors. Licensee MDPI, Basel, Switzerland. This article is an open access article distributed under the terms and conditions of the Creative Commons Attribution (CC BY) license (http://creativecommons.org/licenses/by/4.0/). 